\title{
TRANSMUTED HALFNORMAL DISTRIBUTION: PROPERTIES AND
}

\section{APPLICATION}

\section{AKOMOLAFE ABAYOMI $\mathrm{A}^{1}$ and MARADESA ADELEKE ${ }^{2}$}

${ }^{1}$ Lecturer, Department of Statistics, Federal University of Technology, Akure, Nigeria. [akomolafe01@yahoo.com]

${ }^{2}$ Research student, Department of Statistics, Federal University of Technology, Akure, Nigeria[maradprime1@gmail.com]

\begin{abstract}
In this research, we generalize the Half normal distribution which is a special case of folded normal and truncated normal distributions. We provide a comprehensive description of the statistical properties of the subject distribution along with its reliability behavior. The usefulness of the transmuted Half normal distribution for modelling reliability data is illustrated.
\end{abstract}

Keywords: Half normal Distribution, Hazard Rate function, Reliability function, Order Statistics and Parameter Estimation

DOI: $10.7176 / \mathrm{MTM} / 9-1-02$

\subsection{Introduction}

The quality of the procedure used in a statistical analysis depends heavily on the assumed probability models or distribution, because of this, considerable efforts has been expended in the development of large classes of standard probability distribution along with relevant statistical methodology, However, there still remain many important problems where the real data does not follow any of the classical or standard probability models. In this research, we present a new generalization of the Half Normal distribution called Transmuted Half Normal Distribution.

\subsection{Transmuted Half Normal Distribution}

The half-normal distribution is a special case of the folded normal and truncated normal distributions. It was used to model brownian movement and can also be used in the modeling measurement data and lifetime data. Let $\mathrm{X} \sim \mathrm{N}\left(0, \sigma^{2}\right)$, then $\mathrm{Y}=|\mathrm{X}|$ follows half normal distribution. The Half-normal is a fold at the mean of an ordinary normal distribution with mean zero, where $\sigma$ is the scale parameter. 
$\mathrm{f}(x)=\frac{\sqrt{2}}{\sigma \sqrt{\pi}} e^{-\frac{x^{2}}{2 \sigma^{2}}}$

From (46), we can obtain the cdf of Halfnormal distribution by using the change of variables and let $\mathrm{y}=\frac{x^{2}}{2 \sigma^{2}} ; y=\frac{x}{\sigma \sqrt{2}} \quad ; \mathrm{d} x=\frac{1}{\sigma \sqrt{2}} d y$; which can be $\mathrm{d} x=\sigma \sqrt{2} d y \quad$ by cross multiplication. Now substitute for $\mathrm{y}$ and $\mathrm{d} x$ in

$\mathrm{F}(\mathrm{y} ; \quad \sigma \quad)$ $=$

$\int_{0}^{t} \frac{\sqrt{2}}{\sigma \sqrt{\pi}} e^{-y_{\sigma}} \sqrt{2} d y=\int_{0}^{t} \frac{2}{\sqrt{\pi}} e^{-y^{2}} d y=\operatorname{erf}(y)=\operatorname{erf}\left(\frac{y}{\sigma \sqrt{2}}\right)-\operatorname{erf}(0) ;$ when $t=\frac{y}{\sigma \sqrt{2}}$

Therefore, the $\mathrm{F}(\mathrm{y} ; \sigma)$ in term of error function is displayed below

$\mathrm{F}_{\mathrm{HND}}(\mathrm{y} ; \sigma)=\operatorname{erf}\left(\frac{y}{\sigma \sqrt{2}}\right)$ or $\mathrm{F}(\mathrm{x} ; \sigma)=\operatorname{erf}\left(\frac{x}{\sigma \sqrt{2}}\right)$

$\mathrm{F}(\mathrm{x})=\operatorname{erf}\left(\frac{x}{\sigma \sqrt{2}}\right)$

The (1) and (2) above are respectively the pdf and cdf of halfnormal distribution. The transmuted distribution can be obtained by adding a real number $\lambda$ to the cumulative distribution. That is, if there a cdf $\mathrm{G}(\mathrm{x})$ of any random variable $\mathrm{X}$, then the function (3) is called the transmuted distribution (Shaw and Buckley, 2007)

$\mathrm{F}(\mathrm{x})=(1+\lambda) G(x)-\lambda(\mathrm{G}(\mathrm{x}))^{2}$ where $|\lambda| \leq 1$

From (48) by substituting (47) in (48) we obtain the cdf of transmuted halfnormal distribution.

$\mathrm{F}(\mathrm{x}, \sigma, \lambda)=,(1+\lambda)\left(\operatorname{erf}\left(\frac{x}{\sigma \sqrt{2}}\right)\right)-\lambda\left(\operatorname{erf}\left(\frac{x}{\sigma \sqrt{2}}\right)\right)^{2}$

$=f(x)=\frac{d}{d x}\left[(1+\lambda) G(x)-\lambda(\mathrm{G}(\mathrm{x}))^{2}\right]$

$=(1+\lambda) g(x)-2 \lambda \mathrm{g}(\mathrm{x}) \mathrm{G}(\mathrm{x})$

Substitute for pdf and cdf of the parent distribution (Halfnormal distribution) in (108), then

$$
\begin{aligned}
& \mathrm{f}(\mathrm{x})=(1+\lambda) \frac{\sqrt{2}}{\sigma \sqrt{\pi}} e^{-\frac{x^{2}}{2 \sigma^{2}}}-2 \lambda \frac{\sqrt{2}}{\sigma \sqrt{\pi}} e^{-\frac{x^{2}}{2 \sigma^{2}}} \cdot \operatorname{erf}\left(\frac{x}{\sigma \sqrt{2}}\right) \\
& \mathrm{f}(\mathrm{x})=\left[(1+\lambda)-2 \lambda \operatorname{erf}\left(\frac{x}{\sigma \sqrt{2}}\right)\right] \frac{\sqrt{2}}{\sigma \sqrt{\pi}} e^{-\frac{x^{2}}{2 \sigma^{2}}}
\end{aligned}
$$

The (6) above is the pdf of Transmuted HalfNormal distribution

\subsection{Hazard Rate Function}

$=\boldsymbol{h}(\boldsymbol{x} ; \lambda, \sigma)=\frac{[1+\lambda-2 \lambda \mathrm{G}(\mathrm{x})] \boldsymbol{g}(\boldsymbol{x})}{[1-G(x)][1-\lambda \mathrm{G}(\mathrm{x})]}=\boldsymbol{h}_{G}(\boldsymbol{x} ; \sigma) \frac{[1+\lambda-2 \lambda \mathrm{G}(\mathrm{x})]}{1-\lambda \mathrm{G}(\mathrm{x})}$ where $\boldsymbol{h}_{G}(\boldsymbol{x} ; \sigma)$ is the baseline (parent) distribution.

$$
=\boldsymbol{h}(\boldsymbol{x} ; \lambda, \sigma)=\frac{\frac{\sqrt{2}}{\sigma \sqrt{\pi}} e^{-\frac{x^{2}}{2 \sigma^{2}}}}{1-\operatorname{erf}\left(\frac{x}{\sigma \sqrt{2}}\right)} \cdot \frac{1+\lambda-2 \lambda \cdot \operatorname{erf}\left(\frac{x}{\sigma \sqrt{2}}\right)}{1-\lambda \operatorname{erf}\left(\frac{x}{\sigma \sqrt{2}}\right)}=\frac{\left[1+\lambda-2 \lambda \operatorname{erf}\left(\frac{x}{\sigma \sqrt{2}}\right)\right] \frac{\sqrt{2}}{\sigma \sqrt{\pi}} e^{-\frac{x^{2}}{2 \sigma^{2}}}}{\left[1-\operatorname{erf}\left(\frac{x}{\sigma \sqrt{2}}\right)\right]\left[1-\lambda \operatorname{erf}\left(\frac{x}{\sigma \sqrt{2}}\right)\right]}
$$


asymtotic of $\mathbf{f}(\mathbf{x} ; \lambda, \sigma)$ AND $\mathbf{F}(\mathbf{x} ; \lambda, \sigma)$ as $\boldsymbol{G}(\boldsymbol{x}) \rightarrow \mathbf{0}$

$\mathrm{F}(\mathrm{x}) \sim(1+\lambda) G(x), \quad f(x) \sim(1+\lambda) g(x), \quad h(x) \sim(1+\lambda) /(1-G(x))$

asymtotic of $\mathbf{f}(\mathbf{x} ; \lambda, \sigma)$ AND $\mathbf{F}(\mathbf{x} ; \lambda, \sigma)$ as $\quad \boldsymbol{x} \rightarrow \mathbf{0}$

1-F(x) $\sim \mathrm{G}(\mathrm{x})[\mathrm{G}(\mathrm{x})-1], \mathrm{f}(\mathrm{x}) \sim(1+\lambda) \mathrm{g}(\mathrm{x}), h(x) \sim(1+\lambda) /(1-G(x))$

\subsection{Order Statistics}

According to Marcelo Bourguignon et al (2016), the order statistics for transmuted family can be obtained by (110).

$=f_{r . n}(x ; \lambda, \sigma)=\frac{1}{B(r, n-r+1)} F(x)^{r-1}[1-F(x)]^{n-r}$ which can be view as (7) when defining it in term of transmuted family using the required baseline (parent) distribution.

$=$

$\frac{1}{B(r, n-r+1)} \sum_{j=0}^{n-r}(-1)^{j}\left(\begin{array}{c}n-j \\ j\end{array}\right)\left[(1+\lambda) G(x ; \sigma)-\lambda(\mathrm{G}(x ; \sigma))^{2}\right]^{r-1+j} \cdot[1+\lambda-$ $2 \lambda \mathrm{G}(x ; \sigma)] g(x)$

(7)

By substituting the pdf and cdf of parent distribution we obtain the order statistics of transmuted halfnormal distribution (THND) .

$=$

$\frac{1}{B(r, n-r+1)} \sum_{j=0}^{n-r}(-1)^{j}\left(\begin{array}{c}n-j \\ j\end{array}\right)\left[(1+\lambda) \operatorname{erf}\left(\frac{x}{\sigma \sqrt{2}}\right)-\left(\operatorname{erf}\left(\frac{x}{\sigma \sqrt{2}}\right)\right)^{2}\right]^{r-1+j}[1+\lambda-$ $\left.2 \lambda \operatorname{erf}\left(\frac{x}{\sigma \sqrt{2}}\right)\right] \frac{\sqrt{2}}{\sigma \sqrt{\pi}} e^{-\frac{x^{2}}{2 \sigma^{2}}}$

The $\mathrm{k}^{\text {th }}$ order moment of $X_{r, n}$ is displayed (113) below:

$=\int_{0}^{\infty} x^{k}\left[\left(1+\lambda \mathrm{G}(\mathrm{x} ; \sigma)-\lambda(\mathrm{G}(\mathrm{x}))^{2}\right)\right]^{r+j-1}[1+\lambda-2 \lambda \mathrm{G}(\mathrm{x} ; \sigma)] g(x ; \sigma)$

The (9) can be simply by binomial expansion as (10)

$=\frac{1}{B(r, n-r+1)} \sum_{j=0}^{n-r}(-1)^{j}\left(\begin{array}{c}n-r \\ j\end{array}\right) J$

$\mathrm{J}=\int_{0}^{1} \frac{1}{G(x ; \sigma)} \cdot(1+\lambda-2 \lambda \mathrm{t}) \cdot\left[(1+\lambda) t-\lambda \mathrm{t}^{2}\right]^{r+j-1}$, substitute for $\mathrm{G}(\mathrm{x} ; \sigma)$ in $\mathrm{J}$ to obtain the $\mathrm{k}^{\text {th }}$ order moment of Transmuted Half-Normal.

$=I=\int_{0}^{1} \frac{1}{\operatorname{erf}\left(\frac{x}{\sigma \sqrt{2}}\right)} \cdot(1+\lambda-2 \lambda \mathrm{t}) \cdot\left[(1+\lambda) t-\lambda \mathrm{t}^{2}\right]^{r+j-1}$. Put for $\mathrm{J}$ in equation (11).

$=\frac{1}{B(r, n-r+1)} \sum_{j=0}^{n-r}(-1)^{j}\left(\begin{array}{c}n-r \\ j\end{array}\right) \int_{0}^{1} \frac{1}{\operatorname{erf}\left(\frac{x}{\sigma \sqrt{2}}\right)} \cdot(1+\lambda-2 \lambda \mathrm{t}) \cdot\left[(1+\lambda) t-\lambda \mathrm{t}^{2}\right]^{r+j-1}$

$\mathrm{J}$ can be evaluated using numerical integration.

\subsection{Probability Weighted Moment}


According to Marcelo Bourguignon et al (2016), the probability weighted moment for transmuted family can be obtained by

$=W_{n . r}=\int_{-\infty}^{\infty} x^{n}\left[(1+\lambda) G(x ; \sigma)-\lambda(\mathrm{G}(\mathrm{x}, \sigma))^{2}\right] d x$

using binomial expansion, the result can be rewritten

$$
\begin{aligned}
& W_{n, r}=\sum_{j=0}^{r}(-\lambda)^{j}(1+\lambda)^{r-j}\left(\begin{array}{l}
r \\
j
\end{array}\right)\left[(1+\lambda) \tau_{n, r-j}-2 \lambda \tau_{n, \mathrm{r}+1-\mathrm{j}}\right] \\
& \tau_{n, r-j}=\int_{-\infty}^{\infty} x^{n}[G(x)]^{r-j} d x=\int_{-\infty}^{\infty} x^{n}\left[\operatorname{erf}\left(\frac{x}{\sigma \sqrt{2}}\right)\right]^{r-j} d x
\end{aligned}
$$

From (59) let $\mathrm{y}=\frac{x}{\sigma \sqrt{2}}, \frac{d y}{d x}=\frac{1}{\sigma \sqrt{2}}$, then $d x=d x=\sigma \sqrt{2} d y$

$=\int_{-\infty}^{\infty} x^{n}[\operatorname{erf}(y)]^{r-j} d x=\int_{-\infty}^{\infty}(\sigma y \sqrt{2})^{n}[\operatorname{erf}(y)]^{r-j} \sigma \sqrt{2} d y$

$\tau_{n, r-j}=(\sigma \sqrt{2})^{n+1} \int_{-\infty}^{\infty} y^{n}[\operatorname{erf}(y)]^{r-j} d y$

$=\tau_{n, r-j+1}=(\sigma \sqrt{2})^{n+1} \int_{-\infty}^{\infty} y^{n}[\operatorname{erf}(y)]^{r-j+1} d y$

The (16) and (17) can be obtained by following some numerical method of integration and substitute them in (14) to obtain the Probability weighted moment.

\subsection{Maximum Likelihood Method for THND}

We consider the estimation of parameters of Transmuted family from samples by maximum likelihood. Let $x_{1}, x_{2} \ldots x_{n}$ be observed values from this family with parameter $\theta=\lambda, \sigma$.

$$
\begin{aligned}
& =l(\theta)=\sum_{i=1}^{n} \log \left[g(x ; \sigma)+\sum_{i=1}^{n}[1+\lambda-2 \lambda \mathrm{G}(\mathrm{x}, \sigma)]\right] \\
& =\sum_{i=1}^{n} \log \left[\frac{\sqrt{2}}{\sigma \sqrt{\pi}} e^{-\frac{x^{2}}{2 \sigma^{2}}}\right\rceil+\sum_{i=1}^{n}\left[1+\lambda-2 \lambda \operatorname{erf}\left(\frac{x}{\sigma \sqrt{2}}\right)\right]
\end{aligned}
$$

To obtain the information matrix, we follow this procedure.

$$
\begin{aligned}
& =u_{\lambda}=-2 \sum_{i=1}^{n}\left[\frac{\mathrm{G}(\mathrm{x}, \sigma)}{1+\lambda-2 \lambda \mathrm{G}(\mathrm{x}, \sigma)}\right]=-2 \sum_{i=1}^{n}\left[\frac{\operatorname{erf}\left(\frac{x}{\sigma \sqrt{2}}\right)}{1+\lambda-2 \lambda \operatorname{erf}\left(\frac{x}{\sigma \sqrt{2}}\right)}\right] \\
& =u_{\sigma k}=\sum_{i=1}^{n}\left[\frac{\frac{d g(x ; \sigma)}{d \sigma}}{g(x ; \sigma)}\right]-2 \lambda \sum_{\mathrm{i}=1}^{\mathrm{n}}\left[\frac{\frac{d g(x ; \sigma)}{d \sigma}}{1+\lambda-2 \lambda \mathrm{G}(\mathrm{x}, \sigma)}\right] \\
& =u_{\sigma k}=\sum_{i=1}^{n}\left[\frac{\frac{-\sqrt{2}\left(\sigma^{2}-x^{2}\right)}{\sigma^{4} \sqrt{\pi}} e^{-\frac{x^{2}}{2 \sigma^{2}}}}{\frac{\sqrt{2}}{\sigma \sqrt{\pi}} e^{-\frac{x^{2}}{2 \sigma^{2}}}}\right]-2 \lambda \sum_{\mathrm{i}=1}^{\mathrm{n}}\left[\frac{\frac{-\sqrt{2}\left(\sigma^{2}-x^{2}\right)}{\sigma^{4 \sqrt{\pi}} e^{-\frac{x^{2}}{2 \sigma^{2}}}}}{1+\lambda-2 \lambda\left(\operatorname{erf}\left(\frac{x}{\sigma \sqrt{2}}\right)\right)}\right] \\
& =u_{\lambda \lambda}=-2 \sum_{i=1}^{n} \frac{G\left(x_{i}, \sigma\right)^{2}}{[1+\lambda-2 \lambda \mathrm{G}(\mathrm{x}, \sigma)]^{2}}=-2 \sum_{i=1}^{n} \frac{\left(\operatorname{erf}\left(\frac{x}{\sigma \sqrt{2}}\right)\right)^{2}}{\left[1+\lambda-2 \lambda \operatorname{erf}\left(\frac{x}{\sigma \sqrt{2}}\right)\right]^{2}}
\end{aligned}
$$


$=u_{\lambda \sigma_{1}=-2(1+\lambda)} \sum_{i=1}^{n} \frac{\frac{d g(x ; \sigma)}{d \sigma}}{[1+\lambda-2 \lambda \mathrm{G}(\mathrm{x}, \sigma)]^{2}}=-2(1+\lambda) \sum_{i=1}^{n} \frac{\frac{-\sqrt{2}\left(\sigma^{2}-x^{2}\right)}{\sigma^{4 \sqrt{\pi}}} e^{-\frac{x^{2}}{2 \sigma^{2}}}}{\left[1+\lambda-2 \lambda \operatorname{erf}\left(\frac{x}{\sigma \sqrt{2}}\right)\right]^{2}}$

$=$

$\left[\sum_{i=1}^{n} \frac{g_{k l}{ }^{\prime \prime}\left(x_{i}, \sigma\right) g\left(\left(x_{i}, \sigma\right)\right)-g_{k}{ }^{\prime}\left(x_{i}, \sigma\right) \cdot g_{l}{ }^{\prime}\left(x_{i}, \sigma\right)}{g^{2}\left(\left(x ; \lambda_{i}, \sigma_{k}\right)\right)}-2 \lambda \sum_{\mathrm{i}=1}^{\mathrm{n}} \frac{\mathrm{G}_{\mathrm{kl}}{ }^{\prime \prime}\left(x_{i}, \sigma\right)}{1+\lambda-2 \lambda \mathrm{G}(\mathrm{x}, \sigma)}-\right.$

$\left.4 \lambda \sum_{\mathrm{i}=1}^{\mathrm{n}} \frac{\mathrm{G}_{\mathrm{k}}{ }^{\prime}\left(x ; \lambda_{i}, \sigma_{k}\right) \cdot \mathrm{G}_{1}{ }^{\prime}\left(x ; \lambda_{i}, \sigma_{k}\right)}{[1+\lambda-2 \lambda \mathrm{G}(\mathrm{x}, \sigma)]^{2}}\right]$

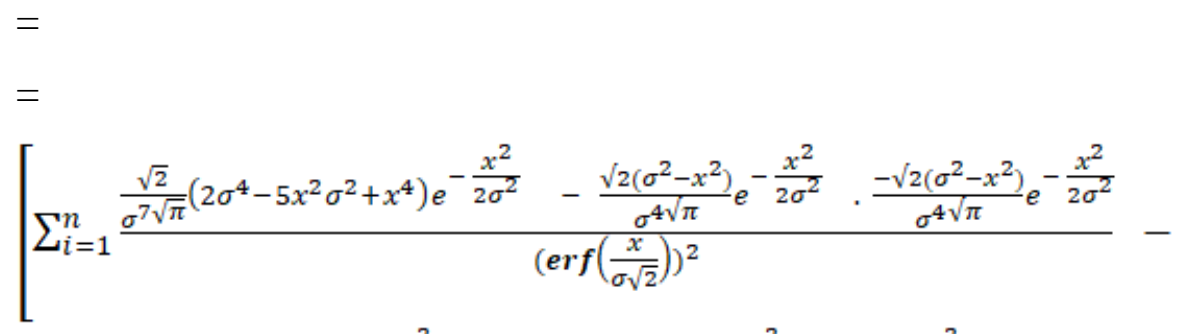

$$
\begin{aligned}
& \left.2 \lambda \sum_{\mathrm{i}=1}^{\mathrm{n}} \frac{\frac{\sqrt{2} \mathrm{x}\left(2 \sigma^{2}-\mathrm{x}^{2}\right) e^{-\frac{x^{2}}{2 \sigma^{2}}}}{\sigma^{5} \sqrt{\pi}}}{1+\lambda-2 \lambda \operatorname{erf}\left(\frac{x}{\sigma \sqrt{2}}\right)}-4 \lambda \sum_{\mathrm{i}=1}^{\mathrm{n}} \frac{\frac{-\sqrt{2} \times e^{-\frac{x^{2}}{2 \sigma^{2}}}}{\sigma^{2} \sqrt{\pi}} \cdot \frac{-\sqrt{2} \mathrm{x} e^{-\frac{x^{2}}{2 \sigma^{2}}}}{\sigma^{2} \sqrt{\pi}}}{\left[1+\lambda-2 \lambda \operatorname{erf}\left(\frac{x}{\sigma \sqrt{2}}\right)\right]^{2}}\right]
\end{aligned}
$$

From $u_{\lambda \lambda}, u_{\lambda \sigma}$ and $u_{\sigma \sigma}$, we can form information matrix called $\mathrm{J}(\theta)$ and the likelihood of the two distributions can be used to test for the goodness of fit to determine if transmuted distribution is superior to the baseline distribution based on the available data. These equations can be solved numerically using Newton-raphson algorithm and the information matrix $\mathbf{J}(\theta)$ is given by (26).

$\mathrm{J}(\theta)=\left(\begin{array}{cc}u_{\lambda \lambda} & u_{\lambda \sigma} \\ u_{\sigma \lambda} & u_{\sigma \sigma}\end{array}\right)$

\section{BETA-HALFNORMAL DISTRIBUTION (BHND)}

Akomolafe et al (2017) used the logit of beta defined by Jones to obtain the mixture of Beta and Half-Normal distribution which we called Beta- Half-Normal distribution. The beta distribution is one of the skewed distributions used in describing uncertainty or random variation in a system. It can be used in modeling complex process due to its multi-faceted structured when varying the parameter values of the distribution. In view of this, The Betahalf-normal distribution was compounded as a mixture of beta and half-normal distribution to give Beta-halfnormal distribution.

$\mathrm{f}_{\mathrm{BHND}}(\mathrm{x} ; \mathrm{a}, \mathrm{b}, \sigma)=\frac{1}{B(a, b)}\left[\operatorname{erf}\left(\frac{x}{\sigma \sqrt{2}}\right)\right]^{a-1}\left[1-\operatorname{erf}\left(\frac{x}{\sigma \sqrt{2}}\right)\right]^{b-1} \frac{\sqrt{2}}{\sigma \sqrt{\pi}} e^{-\frac{x^{2}}{2 \sigma^{2}}}$ 
$\mathrm{F}_{\mathrm{BHND}}(z)=\frac{Z^{a}}{B(a, b)}\left[\frac{1}{a}+\frac{1-b}{a+b}+----+\frac{(1-b)(2-b)(n-b) Z^{n}}{n !(a+b)}\right], \mathrm{Z}=\operatorname{erf}\left(\frac{x}{\sigma \sqrt{2}}\right)$

\subsection{Estimation of Parameters}

\section{Log-Likelihood Function}

Cordeiro et. al [2011] gave the log-likelihood function for $\theta=(a, b, c, \tau)$, where $\tau=\sigma$ as

$\mathrm{L}($ $\theta$ $n \log C-n \log [B(a, b)]+\sum_{i=1}^{n} \log f\left(x_{i} ; \tau\right)+(a-1) \sum_{i=1}^{n} \log F\left(x_{i} ; \tau\right)+(b-$ 1) $\sum_{i=1}^{n} \log \left(1-F^{c}\left(x_{i} ; \tau\right)\right)$

The Generalized Beta distribution reduces to the class of Beta generated distribution when $\mathrm{c}=$ 1 . We hereby have $\theta=(a, b, 1, \sigma)$ which can be rewritten as

$\mathrm{L}($ $\theta$

$-n \log [B(a, b)]+\sum_{i=1}^{n} \log f\left(x_{i} ; \tau\right)+(a-1) \sum_{i=1}^{n} \log F\left(x_{i} ; \tau\right)+(b-1) \sum_{i=1}^{n} \log (1-$ $\left.F^{c}\left(x_{i} ; \tau\right)\right)$

$=-\operatorname{nlog}[B(a, b)]$

$\sum_{i=1}^{n} \log \left[\frac{\sqrt{2}}{\sigma \sqrt{\pi}} e^{-\frac{x^{2}}{2 \sigma^{2}}}\right]+(a-1) \sum_{i=1}^{n} \log \left[\operatorname{erf}\left(\frac{x}{\sigma \sqrt{2}}\right)\right]+(b-1) \sum_{i=1}^{n}\left[1-\operatorname{erf}\left(\frac{x}{\sigma \sqrt{2}}\right)\right]$

$=\frac{\partial L(\theta)}{\partial a}=\frac{-n^{\prime}(a)}{\Gamma(a)}+\frac{n^{\prime}(a+b)}{\Gamma(a+b)}+\sum_{i=1}^{n} \log \left[\operatorname{erf}\left(\frac{x}{\sigma \sqrt{2}}\right)\right]$

$=\frac{\partial L(\theta)}{\partial b}=\frac{-n f(a)}{\Gamma(b)}+\frac{n^{\prime}(a+b)}{\Gamma(a+b)}+\sum_{i=1}^{n} \log \left[1-\operatorname{erf}\left(\frac{x}{\sigma \sqrt{2}}\right)\right]$

$\frac{\partial L(\theta)}{\partial \sigma}=\sum_{i=1}^{n}\left[-\frac{1}{\sigma \sqrt{2}}+\frac{x^{2}}{\sigma^{3}}\right]+(a-1) \sum_{i=1}^{n} \frac{x e^{-\frac{x^{2}}{2 \sigma^{2}} \sqrt{\frac{2}{\pi}}}}{\sigma^{2} \operatorname{erf}\left(\frac{x}{\sigma \sqrt{2}}\right)}+(b-1) \sum_{i=1}^{n} \frac{x e^{-\frac{x^{2}}{2 \sigma^{2}}} \sqrt{\frac{2}{\pi}}}{\left.\sigma^{2} \operatorname{erf}\left(\frac{x}{\sigma \sqrt{2}}\right)-1\right)}=$ $=0$

Expressing (29) and (30) in term of digamma function result to (31) and (32)

$=\frac{\partial L(\theta)}{\partial a}=n(\Psi(a+b-\Psi(a)))+\sum_{i=1}^{n} \log \left[\operatorname{erf}\left(\frac{x}{\sigma \sqrt{2}}\right)\right]=0$

$=\frac{\partial L(\theta)}{\partial b}=n(\Psi(a+b-\Psi(b)))+\sum_{i=1}^{n} \log \left[1-\operatorname{erf}\left(\frac{x}{\sigma \sqrt{2}}\right)\right]=0$

The numerical method can be employed in solving the equations (30),(31) and (32) so as to obtain the parameters $\hat{a}, \hat{b}$ and $\hat{\sigma}$. The loglikelihood can be used to determine if betahalfnormal distribution is better than the parent halfnormal distribution and the information matrix can be used to test the hypothesis that $\mathrm{a}=\mathrm{b}=1$.

\section{Maximum A Posteriori Estimation}

When we consider $\sigma$ as a random variable, the bayes convert our belief about the parameter $\sigma$ of Halfnormal distribution (before seeing data) into posterior probability, $\mathrm{P}(\sigma \mid X)$, by using the likelihood function $\mathrm{P}(\mathrm{X} \mid \sigma)$. The maximum a-posteriori (MAP) estimate is defined as: 
$=\sigma=\operatorname{argmax}(\mathrm{P}(\sigma \mid X))=\operatorname{argmax}_{\sigma} \frac{\mathrm{P}(\mathrm{X} \mid \sigma) \cdot \mathrm{P}(\sigma)}{P(X)}$

$\log \mathrm{L}=n \log \sqrt{2}-n \log \sigma-\frac{\sum x^{2}}{2 \sigma^{2}}+\log B(a, b)^{-1}+(a-1) \log \sigma+(b-1) \log (1-\sigma)$

$=\frac{\partial \log L}{\partial \sigma}=-\frac{n}{\sigma}+\frac{\sum x^{2}}{\sigma^{3}}+\frac{(a-1)}{\sigma}+\frac{(b-1)}{(1-\sigma)}=0$

$=\frac{\partial \log L}{\partial a}=\log \sigma+\frac{\partial}{\partial a} \log B(a, b)^{-1}=0$

$=\frac{\partial \log L}{\partial b}=\log (1-\sigma)+\frac{\partial}{\partial b} \log B(a, b)^{-1}=0$

The $\frac{\partial \log L}{\partial a}$ and $\frac{\partial \log L}{\partial b}$ could be further simplify as (141) and (142)

$=\frac{\partial \log L}{\partial a}=\log \sigma+\Psi_{0}(a+b)-\Psi_{0}(b)=0$

$=\frac{\partial \log L}{\partial b}=\log (1-\sigma)+\Psi_{0}(a+b)-\Psi_{0}(a)=0$

Plot of PDF and CDF for Different values of Parameters (BHND, THND and EEPD)

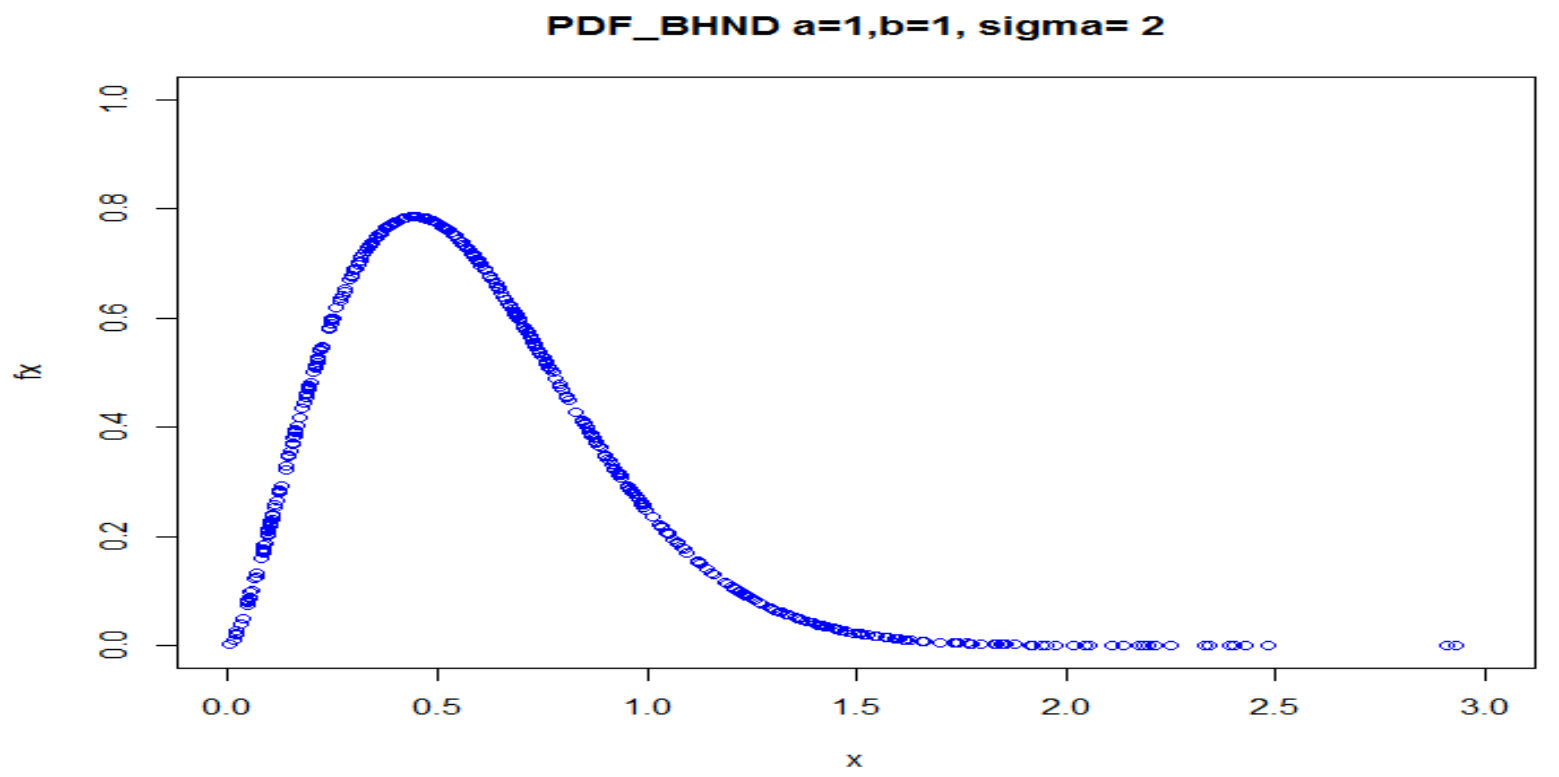

Fig 1: the probability density function of Beta-halfnormal distribution

The fig 1 above shows the probability density of beta-halfnormal distribution with heavy tail to the right. 


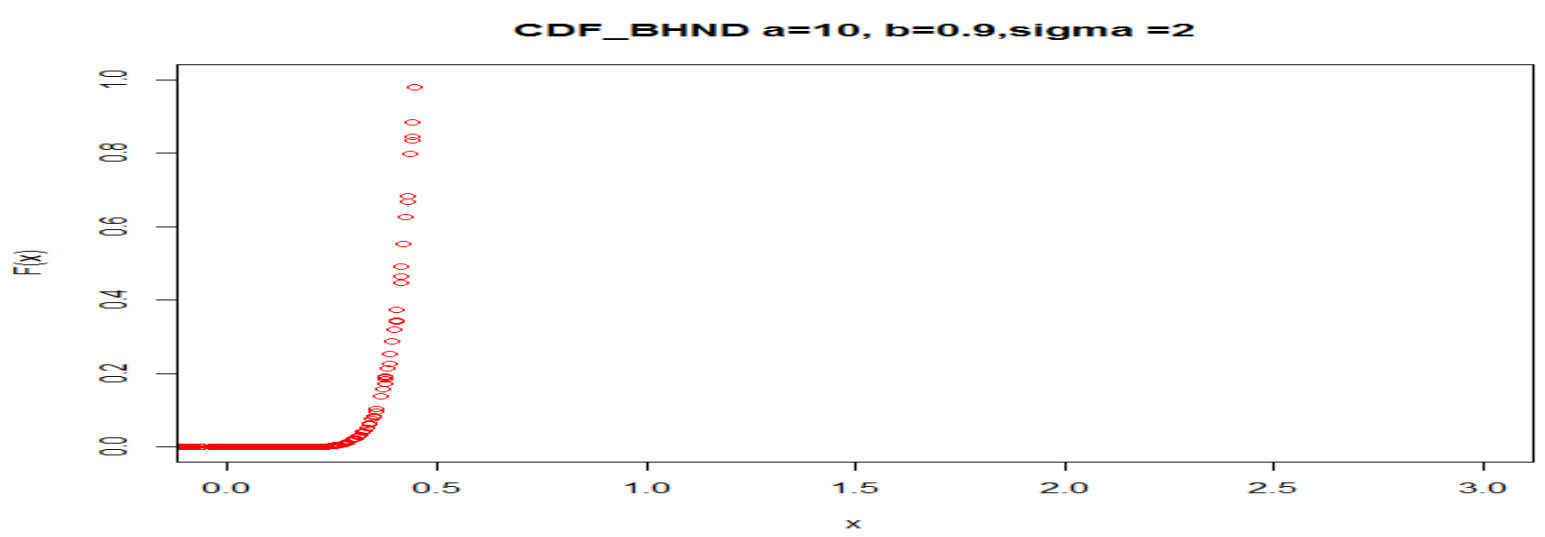

Fig 2: the cumulative distribution function of Beta-halfnormal distribution

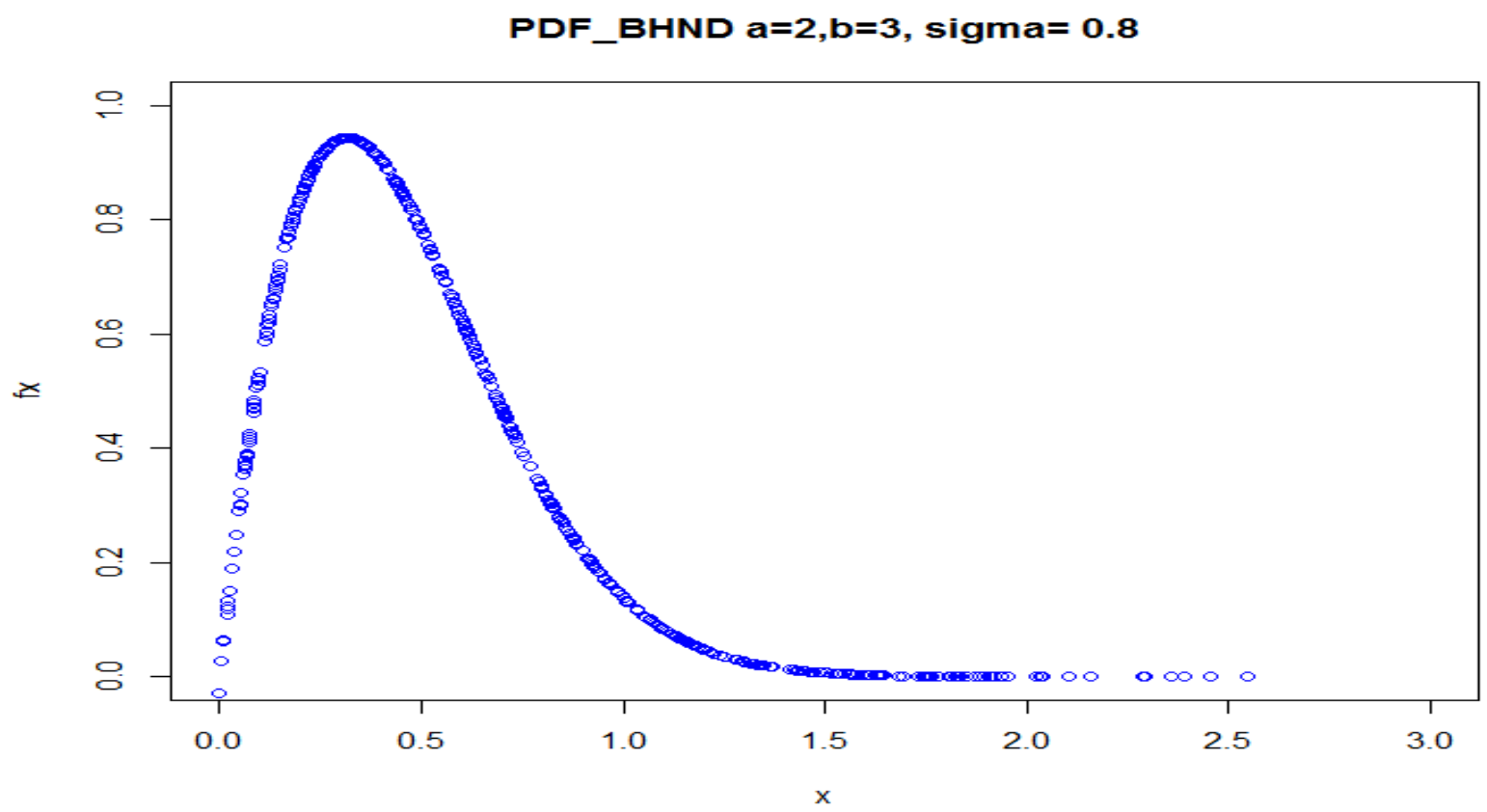

Fig 3: probability density of Beta-halfnormal at $\mathrm{a}=2, \mathrm{~b}=3$ and $\sigma=0.8$

From fig 3, we can say that increased in the value of a and b makes the density plot more kurtic and heavily tailed. 
Pdf_THND lambda=2, sigma $=1$

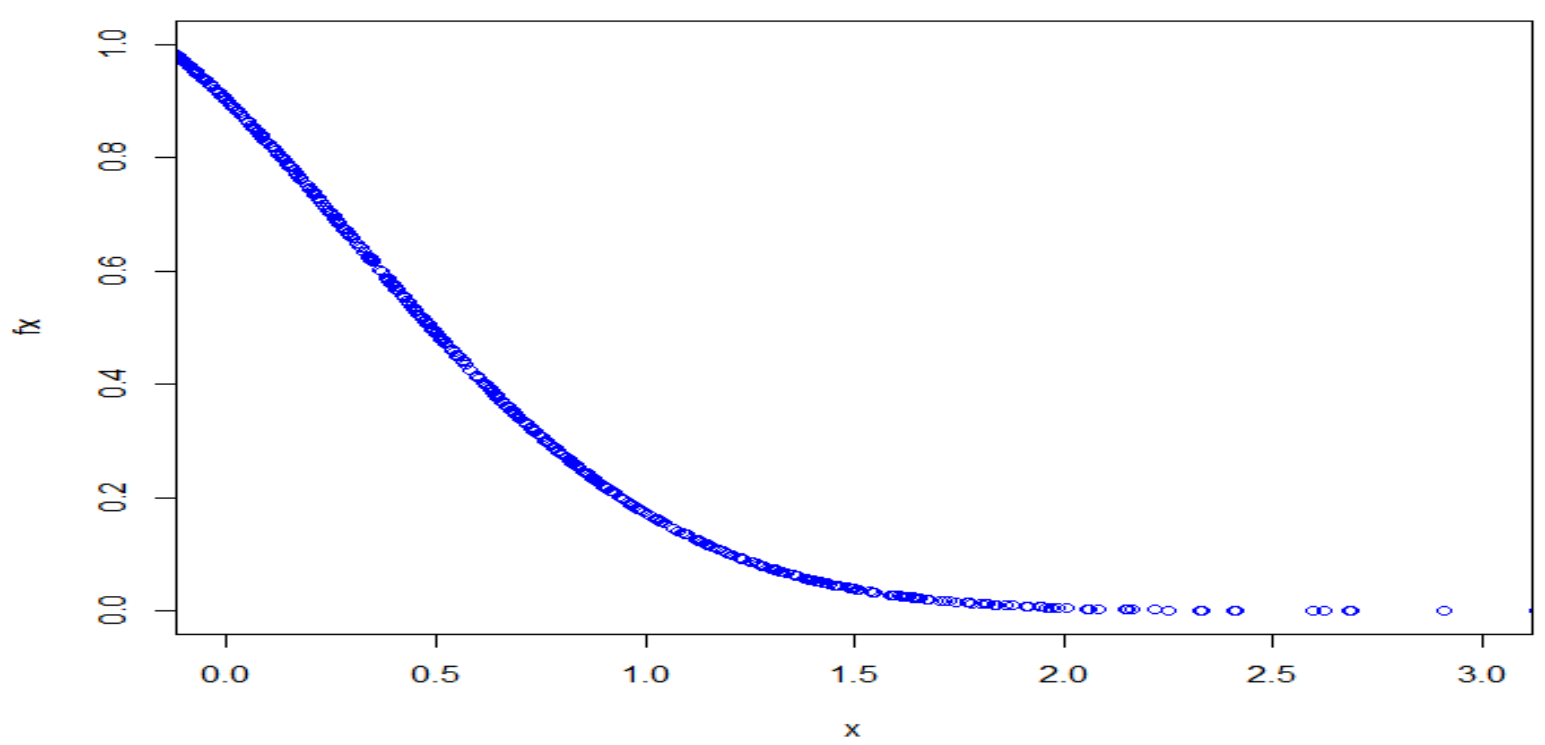

Fig 4: the pdf of transmuted halfnormal distribution (THND)

From fig 4, we can say that the transmuted halfnormal is heavily tailed

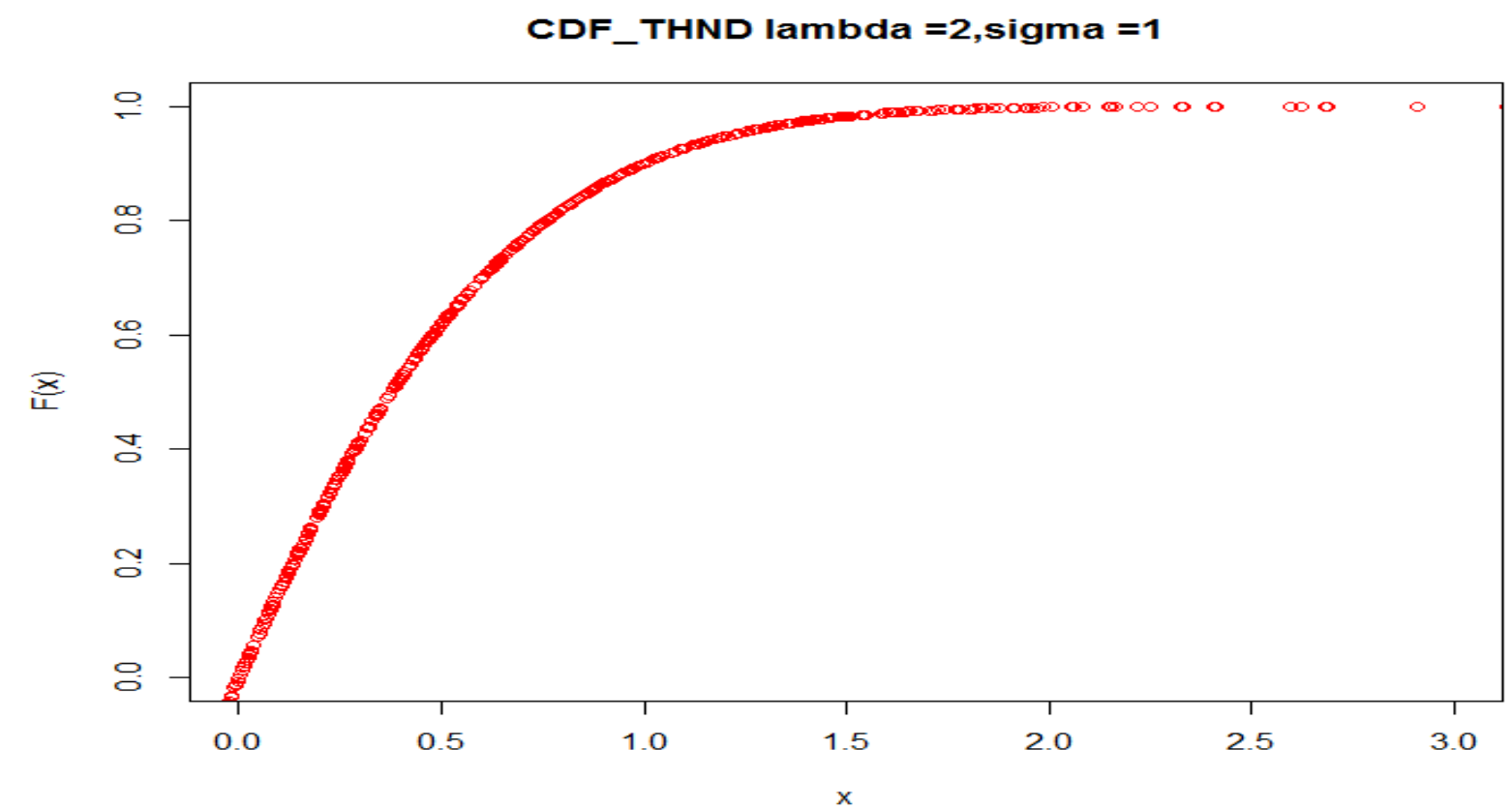

Fig 5: the cumulative distribution function of transmuted halfnormal distribution with $\lambda=2$ and $\sigma=1$ 


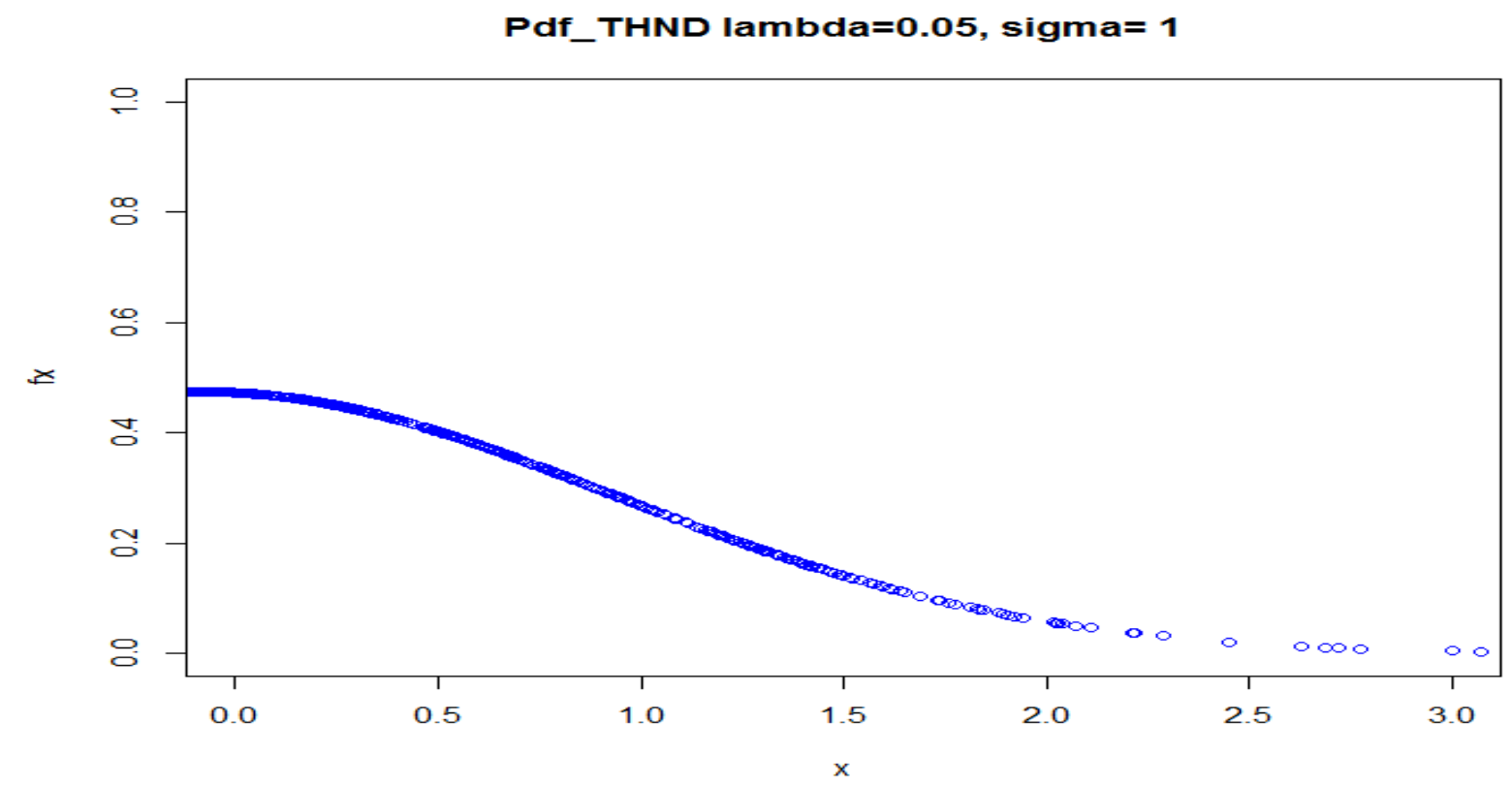

Fig 6: the probability density function $\lambda=0.05$ and $\sigma=1$

From fig 6 , we can say as the value of parameter $\boldsymbol{\lambda}$ reduces $(\boldsymbol{\lambda} \rightarrow \mathbf{0})$, the height of the pdf will reduce making its tail to be heavier.

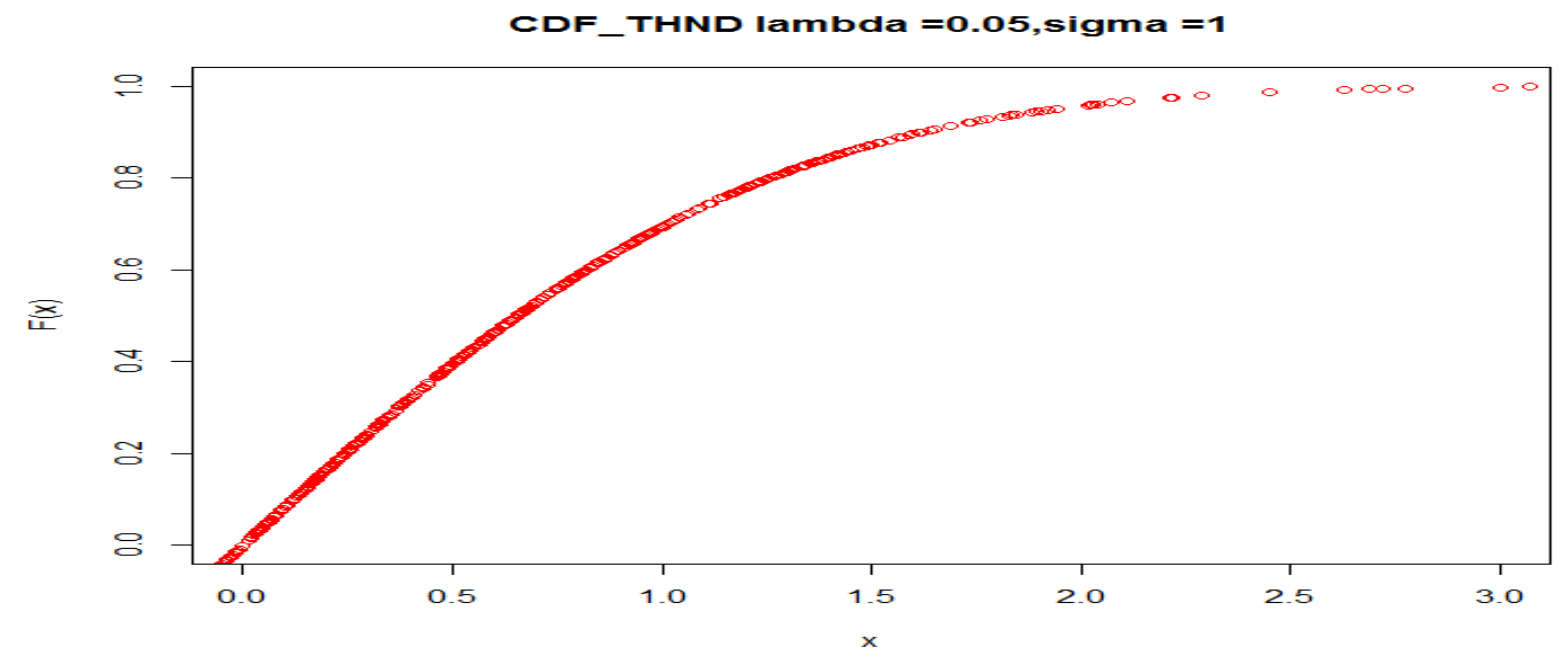

Fig 7: cumulative distribution function of transmute halfnormal $\lambda=0.05$ and $\sigma=1$ 


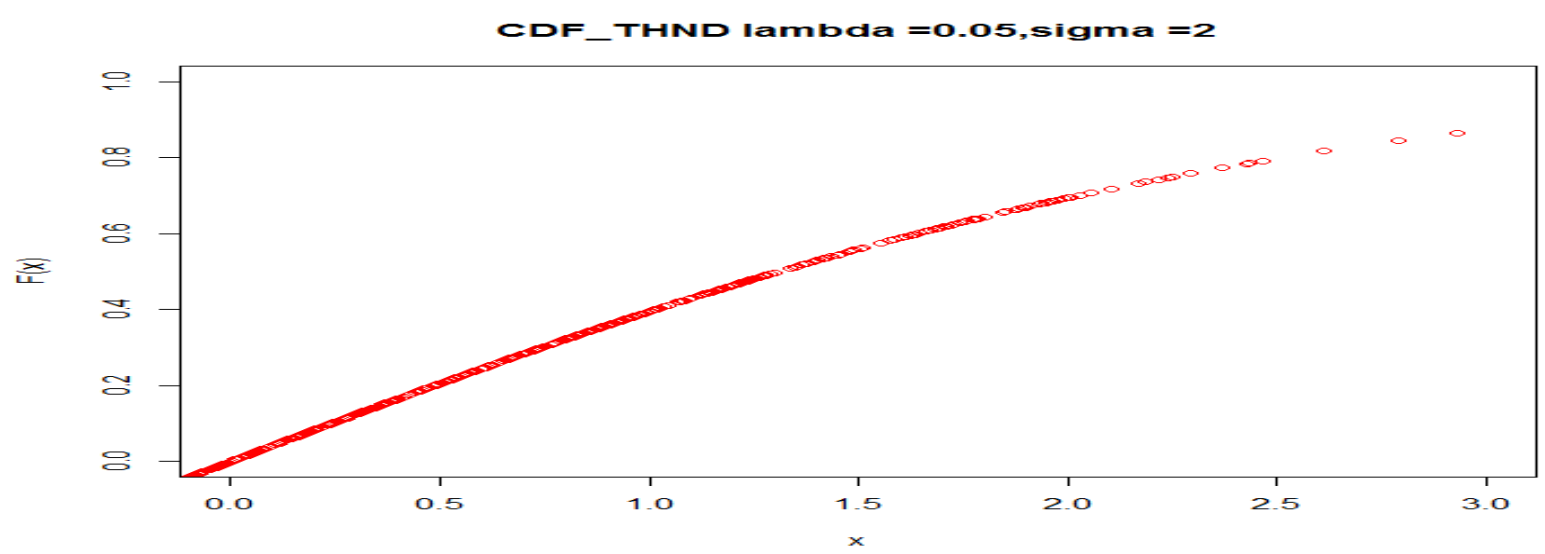

Fig 8: the cumulative distribution function of transmuted half normal $\lambda=0.05$ and $\sigma=2$.

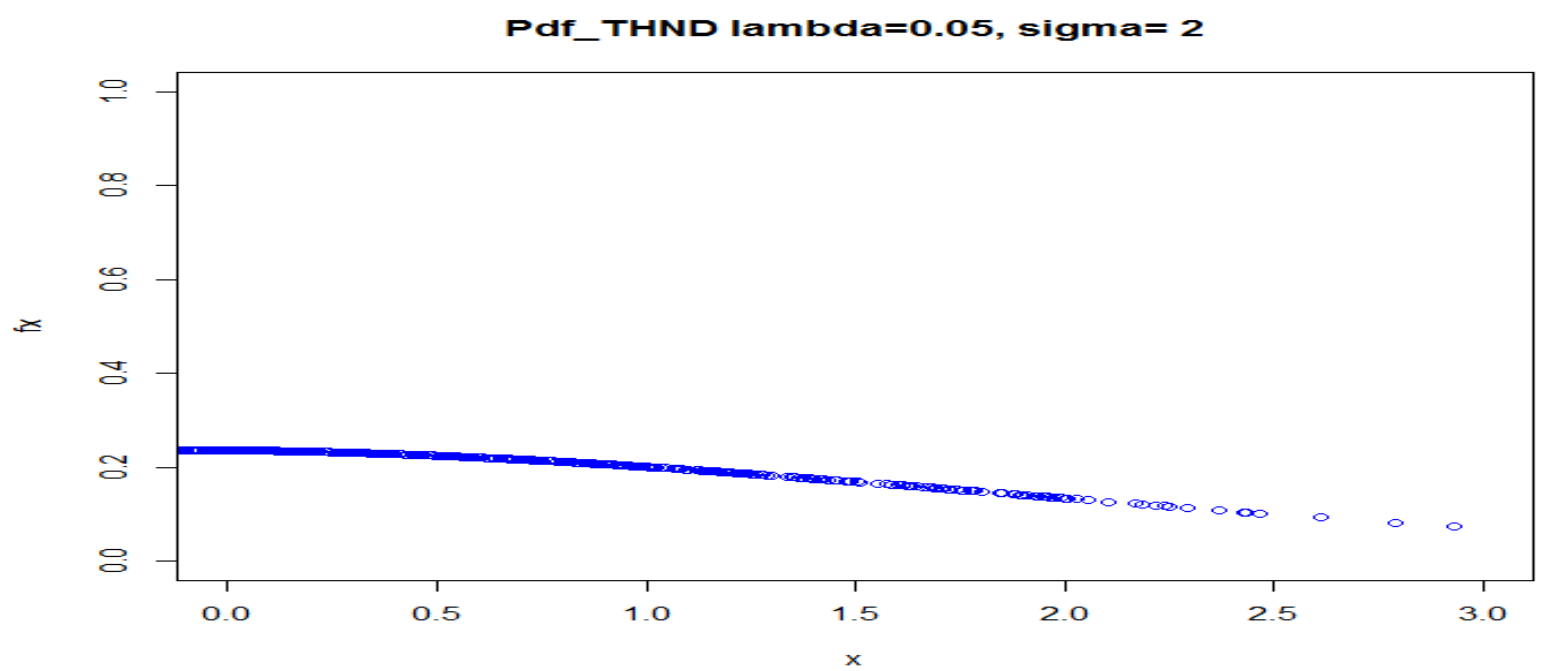

Fig 9: probability density function of transmuted halfnormal distribution $\lambda=0.05$ and $\sigma=2$.

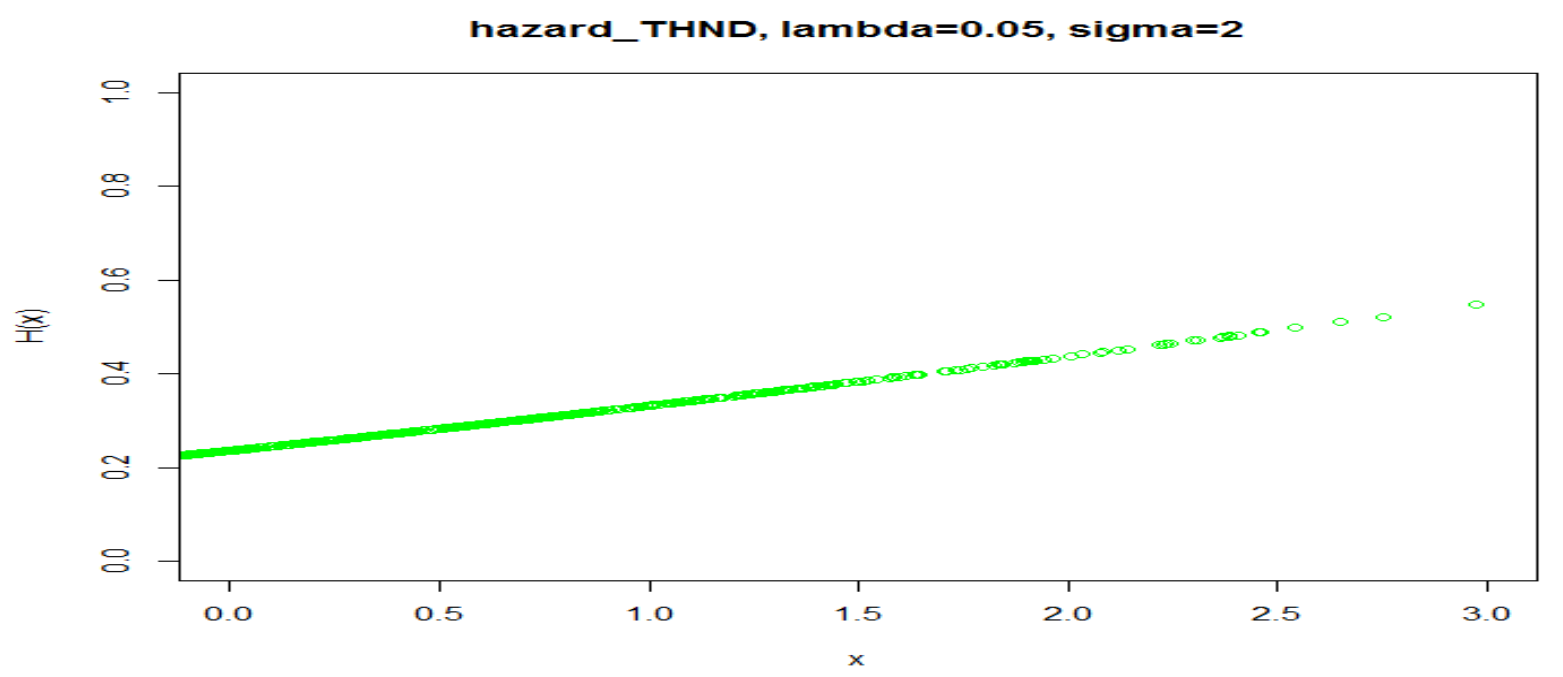

Fig 10: the hazard function of transmuted halfnormal $\lambda=0.05$ and $\sigma=2$. 


\section{APPLICATION}

Now we use a real data set to show that the THND can be a better model than the HND.We work on buying behavior data culled from a standard wholesales outlet, we analyse the data by fitting the hybrid newly developed model as well as the HND on it and the table below shows the parameter estimation.

Table 1: Parameter Estimation by Fitting the Hybrid Models to Buying Behavior Data

\begin{tabular}{|ll|l|l|l|}
\hline parameter & & Estimate & Standard Error & t-value \\
\hline THND & $\lambda$ & 2.124 & 0.212 & 10.23 \\
\hline & $\sigma$ & 1.324 & 0.130 & 9.631 \\
\hline & & & & \\
\hline BHND & $\mathrm{a}$ & 3.213 & 0.829 & 8.342 \\
\hline & $\mathrm{b}$ & 4.541 & 0.932 & 8.763 \\
\hline HND & 1.361 & 0.718 & 7.342 \\
\hline & & 1.921 & & 9.527 \\
\hline
\end{tabular}

\section{WALD TEST}

$\mathrm{H}_{0}: \lambda \neq 0, \alpha=0.05$ (the data supported (THND)) VERSUS $\mathrm{H}_{1}: \lambda=0$

Decision Rule: Accept $\mathrm{H}_{0}$ if $w<\chi_{q}{ }^{2}$, otherwise do not accept

$\mathrm{W}=(\mathrm{R} \hat{\theta}-r)\left(\mathrm{RV}^{\prime}\right)^{-1}(\mathrm{R} \widehat{\theta}-r) \sim \chi_{q}{ }^{2} \quad$ where $\mathrm{q}$ is the number of parameters in the model or the number of rows of the variance-covariance matrix

$=\chi_{q}{ }^{2}$, at $\mathrm{q}=2$, is $0.103 . \mathrm{H}=\left(\begin{array}{cc}-0.0921 & -0.2916 \\ -0.2916 & 0.7207\end{array}\right), \mathrm{w}=0.023$

Decision

Since the $\mathrm{w}<\chi_{q}{ }^{2}$, it establishes reason to accept $\mathrm{H}_{0}$ and conclude that Transmuted halfnormal captures the buying behavior data more than halfnormal distribution because of the increased in number of parameters which control the flexibility of the distribution. 


\section{COMPARISON OF CRITERIA}

In order to compare the two distribution models, we consider the criterion like AIC [ Akaike Information Criterion[,CAIC [Corrected Akaike Information Criterion] and BIC [Bayesian Information Criterion].Transmuted HalfNormal Distribution still establishes that it has a better fit than HalfNormal Distribution based on the values shown in the table below

Table 2: Comparison Criteria

\begin{tabular}{|l|l|l|l|l|}
\hline Hybrid Models & Loglikelihood & AIC & CAIC & BIC \\
\hline THND & 400.5621 & 805.1242 & 833.1242 & 803.8846 \\
\hline HND & 423.2286 & 854.4572 & 882.4972 & 853.2176 \\
\hline
\end{tabular}

\section{Conclusion}

In this paper the Transmuted HalfNormal Distribution has been introduced. Its mathematical properties have been dealt with. Parameters have been estimated using the method of Maximum Likelihood. This distribution has also been applied to real data sets. It has been observed that Transmuted HalfNormal Distribution provides a better fit for buying behavior data as compared with HalfNormal Distribution.

\section{Reference}

[1] Alzaatreh, A. Lee, C. and Famoye, F., (2013).A new method for generating families of continuous distributions. Metron, 71(1), 63-79.

[2] Akinsete, A., Famoye, F., and Lee, C.,(2008). The beta- pareto distribution, Statistics 42(6), 547-563.

[3] Akomolafe A.A and Maradesa A., (2017). Beta-halfnormal Distribution and Its Properties, International journal of Advance Research and Publication vol 1 issued 4 (17-22).

[4] Ashour S.K and Eltehiwy M.A., (2013) . Transmuted Lomax distribution, America Journal of Applied Mathematics and Statistics vol1, 6:121-127.

[5] Badmus, N.I., Ikegwu,M. and Emmanuel, (2013). The Beta-Weighted Weibull Distribution: Some properties and application to Bladder Cancer Data, Applied and Computational Mathematics 2:5.

[6] Barreto-Souza, W., Cordeiro G.M., and Simas A.B., (2011).Some results for beta Frechet distribution. Communications in Stat Statistics Theory \&Methods, 40, 798-811. 
[7] Burr, I.W., (1942). Cumulative frequency functions. Annals of Mathematical Statistics, $13,215-232$.

[8] Brown, M., (2003).Buying or Browsing? An Exploration of shopping Orientations and online Purchase Intention, European Journal of Marketing, 37(11/12),1666-1684.

[9] Blackwell, R.D.,Minar, R.D., and Engel, P.W., (2001). Consumer behavior. New York: Harcourt College publisher.

[10] Cordeiro, G.M., Alexandra, C., Ortega, J.M., Edwin, M.M., and Sarabia, J.M., (2011). Generated distribution ICMA centre. Discusion paper in Finance 1-29.

[11] Eugene, N., Lee, C, and Famoye, F., (2002): Beta-normal distribution and its applications. Communications in Statistic: Theory and methods, 31(4), 497-512.

[12] Famoye, F., Lee, C. and Olumolade, O., (2005). The Beta-Weibull distribution. Journal of Statistical Theory, 4,121-136

[13] Famoye, F., Lee, C. and Eugene, N., (2004). Betanormal distribution: bimodality properties and application. Journal of Modern Applied Statistical Methods, 3, 85-103.

[14] Jones, M.C., (2004). Families of distribution arinsing from distribution of order statistic test $13(1-43)$

[15] Marcelo B., Indranil G., and Cordeiro G.M., (2016), General results for the transmuted Family of Distributions and New Models, Journal of Probability and Statistics. 